\title{
Optical trapping reveals differences in dielectric and optical properties of copper nanoparticles compared to their oxides and ferrites
}

\begin{abstract}
Pablo Purohit ${ }^{1,3}$, Akbar Samadi ${ }^{2,3}$, Poul Martin Bendix ${ }^{2}$, J. Javier Laserna ${ }^{1} \&$ Lene B. Oddershede ${ }^{2 *}$

In a nanoplasmonic context, copper ( $\mathrm{Cu}$ ) is a potential and interesting surrogate to less accessible metals such as gold, silver or platinum. We demonstrate optical trapping of individual Cu nanoparticles with diameters between 25 and $70 \mathrm{~nm}$ and of two ionic Cu nanoparticle species, $\mathrm{CuFe}_{2} \mathrm{O}_{4}$ and $\mathrm{CuZnFe}_{2} \mathrm{O}_{4}$, with diameters of $90 \mathrm{~nm}$ using a near infrared laser and quantify their interaction with the electromagnetic field experimentally and theoretically. We find that, despite the similarity in size, the trapping stiffness and polarizability of the ferrites are significantly lower than those of Cu nanoparticles, thus inferring a different light-particle interaction. One challenge with using Cu nanoparticles in practice is that upon exposure to the normal atmosphere, Cu is spontaneously passivated by an oxide layer, thus altering its physicochemical properties. We theoretically investigate how the presence of an oxide layer influences the optical properties of $\mathrm{Cu}$ nanoparticles. Comparisons to experimental observations infer that oxidation of CuNPs is minimal during optical trapping. By finite element modelling we map out the expected temperature increase of the plasmonic Cu nanoparticles during optical trapping and retrieve temperature increases high enough to change the catalytic properties of the particles.
\end{abstract}

The unique plasmonic properties of metallic nanoparticles have placed them in a privileged spot among other materials in nanoscience ${ }^{1-3}$. Copper nanoparticles (CuNPs) have been proposed for a number of applications within photonics ${ }^{4}$, catalysis $^{5}$, nanothermometry ${ }^{6}$, biosensing ${ }^{7,8}$ or nanomedicine ${ }^{9}$. Nevertheless, the choice of copper tends to fall behind silver, gold or platinum, probably due to its poor stability, i.e., its tendency towards oxidation. Spontaneous formation of Cupric oxide $(\mathrm{CuO})$ and Cuprous oxide $\left(\mathrm{Cu}_{2} \mathrm{O}\right)$ leads to changes of plasmonic properties and chemical reactivity ${ }^{10}$ and is difficult to control. The $\mathrm{Cu}$-containing ferrite $\mathrm{CuFe}_{2} \mathrm{O}_{4}$ (cuprospinel) and $\mathrm{CuZnFe}_{2} \mathrm{O}_{4}$ nanoparticles belong to the spinel group and derive from magnetite ( $\left.\mathrm{Fe} 3 \mathrm{O} 4\right)$ with $\mathrm{Cu}^{+2}$ and $\mathrm{Zn}^{+2}$ partially substituting $\mathrm{Fe}^{+2}$ in the crystalline structure. These particles are also of bio-medical interest since cuprospinel can be used as a cytotoxic agent in cancerous cells ${ }^{11}$ and $\mathrm{CuZnFe}_{2} \mathrm{O}_{4}$ has been proposed as an antibacterial agent ${ }^{12}$. Moreover, cuprospinel is a material of interest in photocatalytic production of hydrogen ${ }^{13}$.

In the aforementioned applications the interaction between the $\mathrm{Cu}$ nanoparticles and the incoming light is of crucial importance and in certain applications a controlled positioning of the particle is needed. Optical tweezers are a well-known tool by which nano- or micro-scopic particles can be manipulated and accurately positioned using a highly focused laser beam ${ }^{14}$. Not only dielectric particles but also plasmonic nanoparticles made of gold, silver or platinum have been reported individually optically trapped using a tightly focused Gaussian laser beam, one of the simplest implementations of optical tweezers ${ }^{15-18}$, and their interaction with electromagnetic field, including the absorption and scattering of the particles, is relatively well understood ${ }^{19-23}$. For copper, however, only larger particles, $1-20 \mu \mathrm{m}$, have been reportedly trapped ${ }^{24}$, and a quantitative description of the interaction between CuNPs and the electromagnetic field is still lacking.

The optical trap exerts a harmonic potential on the trapped particle and determination of the spring constant characterizing the potential provides a way to quantify how the particle interacts with the applied EM field. In the current manuscript we demonstrate optical trapping of $\mathrm{Cu}$ nanoparticles, their oxides and ferrites, and also

${ }^{1}$ Universidad de Málaga, Departamento de Química Analítica, Campus de Teatinos s/n, 29071, Málaga, Spain. ${ }^{2}$ Niels Bohr Institute, University of Copenhagen, Blegdamsvej 17, 2100, Copenhagen, Denmark. ${ }^{3}$ These authors contributed equally: Pablo Purohit and Akbar Samadi. *email: oddershede@nbi.ku.dk 

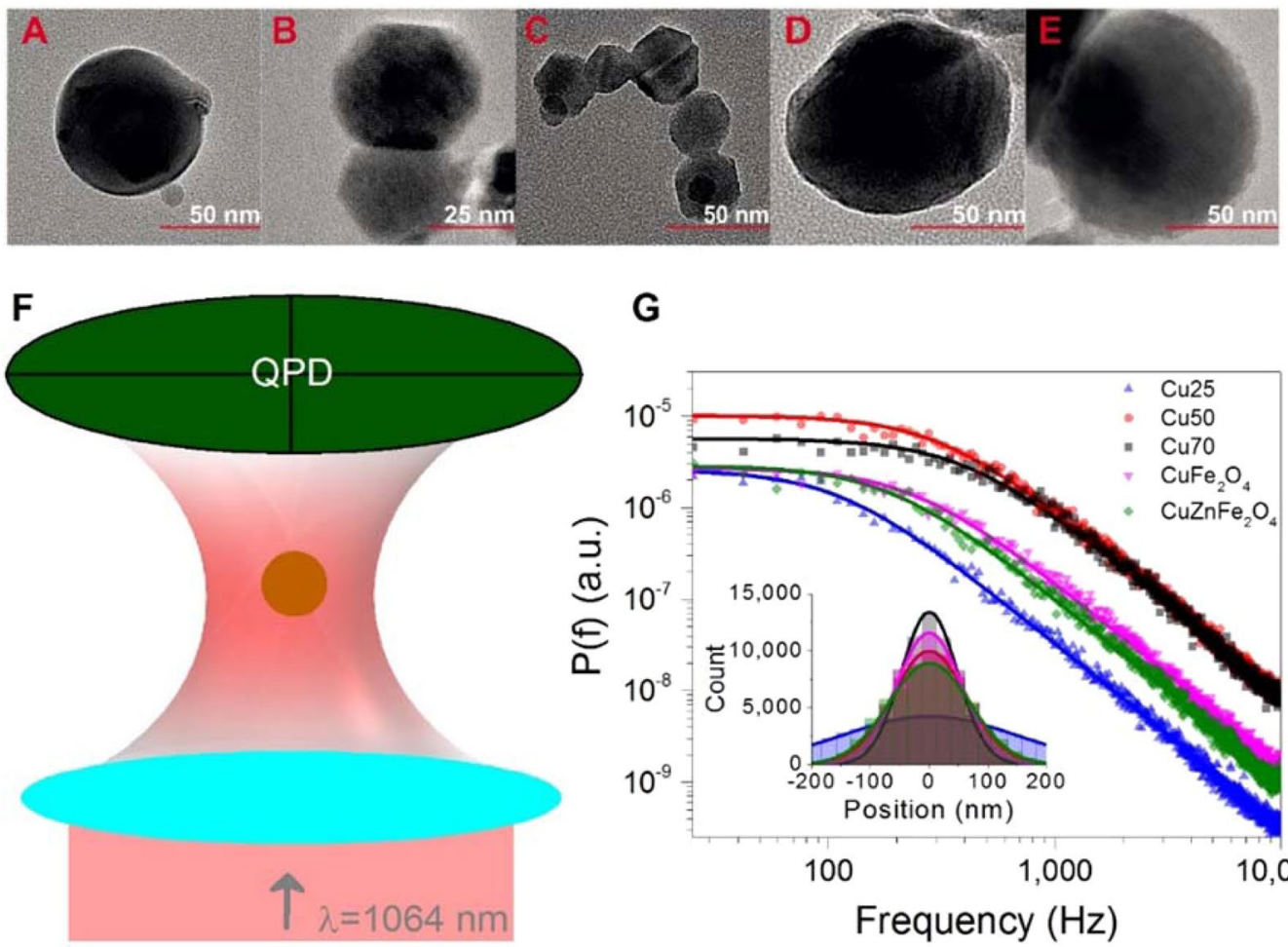

\section{G}

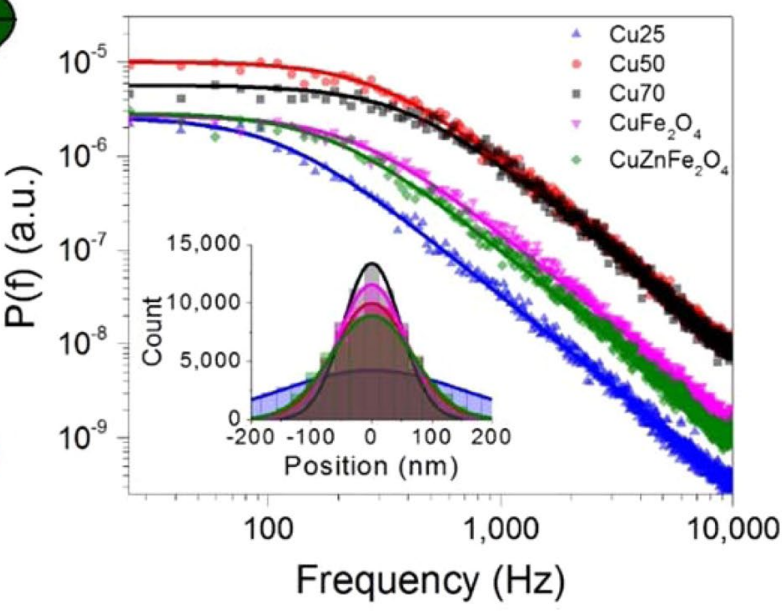

Figure 1. Electron microscopy images of (A) $70 \mathrm{~nm},(\mathbf{B}) 50 \mathrm{~nm}$, (C) $25 \mathrm{~nm}, \mathrm{CuNP}$. (D) $90 \mathrm{~nm} \mathrm{CuFe} \mathrm{O}_{4}$, and (E) $90 \mathrm{~nm} \mathrm{CuZnFe}{ }_{2} \mathrm{O}_{4}$, nanoparticles. (F) Schematic of the optical trapping experiment. (G) Power spectra, $P(f)$, of $\mathrm{Cu} 25$ (blue up-triangles), $\mathrm{Cu} 50$ (red circles), $\mathrm{Cu} 70$ (black squares), $\mathrm{CuFe}_{2} \mathrm{O}_{4}$ (magenta down-triangles) and $\mathrm{CuZnFe}_{2} \mathrm{O}_{4}$ (green diamonds) nanoparticles, trapped by $1064 \mathrm{~nm}$ laser light at $\mathrm{P}=270 \mathrm{~mW}$ at the focal plane. Solid lines depict Lorentzian fits (Eq. 2) of the spectra using routine from ref. ${ }^{25}$. Inset shows positional histograms for the trapped particles along with Gaussian fits (full lines).

use the tweezers to explore and quantify their interaction with the EM field. Our results demonstrate that even smaller changes of chemical bonding within metallic nanoparticles, or the presence of an oxide layer, have substantial effect on the particle's optical properties. Moreover, using Finite Element Modelling (FEM) we calculated relevant optical cross-sections, the polarizability, as well as the expected heating of optically trapped CuNPs. The steep and highly spatially-controlable heating delivered by metallic nanoparticles is of significant interest for catalytic and chemical applications of $\mathrm{CuNPs}^{4}$.

\section{Results and Discussion}

Particle characterization and power spectra acquisition. Diluted suspensions of CuNPs with diameters of $25 \mathrm{~nm}(\mathrm{Cu} 25), 50 \mathrm{~nm}(\mathrm{Cu} 50)$ and $70 \mathrm{~nm}(\mathrm{Cu} 70), 90 \mathrm{~nm}\left(\mathrm{CuFe}_{2} \mathrm{O}_{4}\right)$, and $90 \mathrm{~nm}\left(\mathrm{CuZnFe}_{2} \mathrm{O}_{4}\right)$ were prepared as described in Methods. To evaluate particle morphology and size, TEM images (see equipment details in Methods) of these suspensions were acquired. Example images are shown in Fig. 1A-E and overall, particle sizes and morphologies were found to be in good agreement with those provided by the manufacturer. Figure $1 \mathrm{~F}$ illustrates the experimental setup with which the particles were individually optically trapped by a tightly focused $1064 \mathrm{~nm}$ laser beam. The particle's positional time series was acquired by a quadrant photodiode (QPD). The recordings on the QPD allowed for assessment of whether one or more particles were optically trapped ${ }^{16}$. Due to the high dilution, sonication and filtration of the sample, there was essentially no particle aggregation and most often only a single particle would be trapped; only time series originating from single particle trapping were analysed.

Optical tweezers exert a harmonic force on the trapped NP, $\boldsymbol{F}_{\text {trap }}=-\kappa \boldsymbol{x}$, where $\kappa$ is the spring constant characterizing the optical trap and $\boldsymbol{x}$ is the deviation from the equilibrium position. Hence, if $\kappa$ is known, the force acting on the particle can be found for any position. For this reason, the current manuscript aims at determining $\kappa$ for optical trapping of CuNPs. The particle's dynamics in any translational dimension is well described by the Langevin equation,

$$
m \boldsymbol{a}(\boldsymbol{t})=-\gamma \boldsymbol{v}(\boldsymbol{t})-\kappa \boldsymbol{x}(\boldsymbol{t})+\boldsymbol{F}_{\text {thermal }}(\boldsymbol{t}),
$$

where $m$ is the particle's mass, $\boldsymbol{a}(\boldsymbol{t})$ its acceleration, $\boldsymbol{v}(\boldsymbol{t})$ its velocity, $\gamma$ the friction coefficient, and $\boldsymbol{F}_{\text {thermal }}(\boldsymbol{t})$ is a time-dependent stochastic force originating from thermal collisions within the medium. In water the particle's motion is overdamped and the inertial term, $m \boldsymbol{a}(\boldsymbol{t})$, can be ignored. Hence, the positional power spectrum is given as 


$$
P(f)=\frac{K_{B} T}{\gamma} \frac{1}{f^{2}+f_{c}^{2}},
$$

where $\gamma=6 \pi \eta R$ for spherical particles (with $R$ being the particle's radius and $\eta$ the medium's viscosity) and $f_{c}=\kappa / 2 \pi \gamma$ denotes the corner frequency. Figure $1 \mathrm{G}$ shows power spectra of all trapped particles along with fits (full lines) of Eq. 2 using the routines described in ref. ${ }^{26}$ which return values of $f_{c}$ and hence of $\kappa$, as further discussed in the following section. It is worth noticing that all obtained power spectra are well fitted by Eq. 2 and that the signal is entirely different from that of an empty trap (see Supporting Fig. S1), both in terms of signal amplitude and appearance.

The inset of Fig. 1G demonstrates that all position histograms are Gaussian, as expected because the particle is trapped in a harmonic potential. For pure CuNPs, the width of the histograms, and thereby the variance of the signal, increases with particle size as expected because the smaller the particle, the larger the excursions within the optical trap. For ferrites, the situation is more complex as not only their size but also their material properties change in comparison to the pure CuNPs.

Optical trapping strength of individual CuNPs and ferrites depends on particle size and chemical nature. The trap stiffnesses in the lateral plane $\kappa_{x}$ (parallel to the laser's polarization direction) and $\kappa_{y}$ (orthogonal to the laser's polarization direction) were extracted via power spectral analysis of the time series as described in the preceding section. Values of $\kappa$ versus laser power are shown in Fig. 2A-D. For all particles, the trap stiffness increased linearly with laser power, a hallmark of successful optical trapping. As expected, the larger the solid CuNPs, the higher the spring constant, as observed also for solid gold and silver nanoparticles ${ }^{16,17}$.

The trap stiffnesses of $\mathrm{CuFe}_{2} \mathrm{O}_{4}$ and $\mathrm{CuZnFe}_{2} \mathrm{O}_{4}$ are shown in Fig. 2C,D. In this figure, the lines fitted to the experimentally obtained values of $\kappa$ for $\mathrm{Cu} 70$ and $\mathrm{Cu} 50$ (from Fig. 2A,B) are shown as dashed black and red lines, respectively, to allow for easy comparison. At all laser powers, the trap stiffnesses for the $90 \mathrm{~nm} \mathrm{CuFe}_{2} \mathrm{O}_{4}$ and $\mathrm{CuZnFe}_{2} \mathrm{O}_{4}$ nanoparticles turned out to be lower than those for the $70 \mathrm{~nm}$ CuNPs, despite their larger size. This can be attributed to the ionic chemical bonds which conform the oxides as they restrict the electron mobility through the crystalline structure in stark contrast to the delocalized electron clouds enclosing $\mathrm{Cu}-\mathrm{Cu}$ bonds in the pure metallic particles, thus leading to a more limited particle-laser light interaction for the ferrites.

In order to compare $\kappa$ for $\mathrm{CuZnFe}_{2} \mathrm{O}_{4}$ NPs versus $\kappa$ for $\mathrm{CuFe}_{2} \mathrm{O}_{4} \mathrm{NPs}$ at each laser power (as shown in Fig. 2C,D), we first used a D'Agostino-Pearson's K-squared test to assess the normality of the measured distributions of $f_{c}$ (which are directly proportional to $\kappa$ ) at each laser power. The numbers of individual particles measured at each laser power for $\mathrm{CuFe}_{2} \mathrm{O}_{4}$ and $\mathrm{CuZnFe}_{2} \mathrm{O}_{4}$ are given in Table S1. For each particle, 5 measurements were made, both of $f_{c x}$ and of $f_{c y}$. As all distributions were found to be normally distributed, we proceeded to perform a Two Sample t-test (including Welch's correction for unequal variances) as well as a Two Sample Test for Variance (both with significance level $=0.05$ ), in order to compare the two independent distributions of $f_{c}$ for the two particle types measured at each laser power. For all laser powers, the average value of $f_{c}$ is larger for $\mathrm{CuFe}_{2} \mathrm{O}_{4}$ particles than for $\mathrm{CuZnFe}_{2} \mathrm{O}_{4}$ particles. For laser powers of $223 \mathrm{~mW}$ and above, the two data sets representing $\mathrm{CuFe}_{2} \mathrm{O}_{4}$ and $\mathrm{CuZnFe}_{2} \mathrm{O}_{4} \mathrm{NPs}$, respectively, are significantly different (on a $\mathrm{p}=0.05$ significance level). For example, at a laser power of $\mathrm{P}=223 \mathrm{~mW}$ Welch's t-test returned a value of $\mathrm{p}=0.030$ when comparing $f_{c y}$ values and a value of $\mathrm{p}=0.031$ when comparing $f_{c x}$ values between the two particle types. As $\kappa$ is directly proportional to $f_{c}\left(f_{c}=\kappa / 2 \pi \gamma\right)$, we find that at all laser powers average values of $\kappa$ for $\mathrm{CuFe}_{2} \mathrm{O}_{4}$ particles are larger than for $\mathrm{CuZnFe}_{2} \mathrm{O}_{4}$ particles and for laser powers of $223 \mathrm{~mW}$ and higher, this difference is significant on a 0.05 significance level. This is true both in the $x$ and $y$ directions. A plausible reason for this measured difference in trapping strength could be that the $\mathrm{Zn}-\mathrm{O}$ bond found in $\mathrm{CuZnFe}{ }_{2} \mathrm{O}_{4}$ is more covalent than both the $\mathrm{Cu}-\mathrm{O}$ and $\mathrm{Fe}-\mathrm{O}$ bonds since $\mathrm{Zn}^{+2}$ features a less metallic behavior than the two other counter cations. This leads to an overall lower permittivity, thus explaining the lower trapping efficiency observed for the $\mathrm{CuZnFe}_{2} \mathrm{O}_{4}$ nanoparticles.

Optical cross sections and polarizability of CuNPs. The size of the CuNPs here investigated is small enough that they belong to the Rayleigh regime. In the Rayleigh regime, the optical force can be written as $\boldsymbol{F}=\boldsymbol{F}_{\text {grad }}+\boldsymbol{F}_{\text {scat }}{ }^{26,27}$, where the gradient force, responsible for optical trapping, is given as:

$$
\boldsymbol{F}_{\text {grad }}=\frac{\alpha_{r}}{4} \nabla\left\langle|\boldsymbol{E}|^{2}\right\rangle .
$$

Here, $\alpha_{r}$ is the real part of the polarizability and $\boldsymbol{E}$ is the electric field of the trapping laser.

The scattering force, $\boldsymbol{F}_{\text {scat }}$, destabilizing the optical trap, includes both radiation pressure and spin curl force and is given as:

$$
\boldsymbol{F}_{\text {scat }}=C_{\text {ext }}\left\{\frac{1}{c}\langle\boldsymbol{S}\rangle+c \nabla \times\left\langle\boldsymbol{L}_{\boldsymbol{s}}\right\rangle\right\}
$$

where $C_{\text {ext }}$ is the extinction cross-section of the particle, $\langle\mathbf{S}\rangle$ is the time averaged Poynting vector, $c$ is the speed of light, and $\left\langle\boldsymbol{L}_{\boldsymbol{s}}\right\rangle$ is the time averaged spin density of the electromagnetic field, $\left\langle\boldsymbol{L}_{s}\right\rangle=\frac{\varepsilon_{0}}{4 \omega i}\left\{\boldsymbol{E} \times \boldsymbol{E}^{*}\right\}$. The extinction cross-section is a sum of the scattering and absorption cross-sections: $C_{e x t}=C_{s c a t}+C_{a b s}$.

The particle's complex polarizability, $\alpha=\alpha_{r}+i \alpha_{i}$, is related to its absorption and scattering cross-sections: 

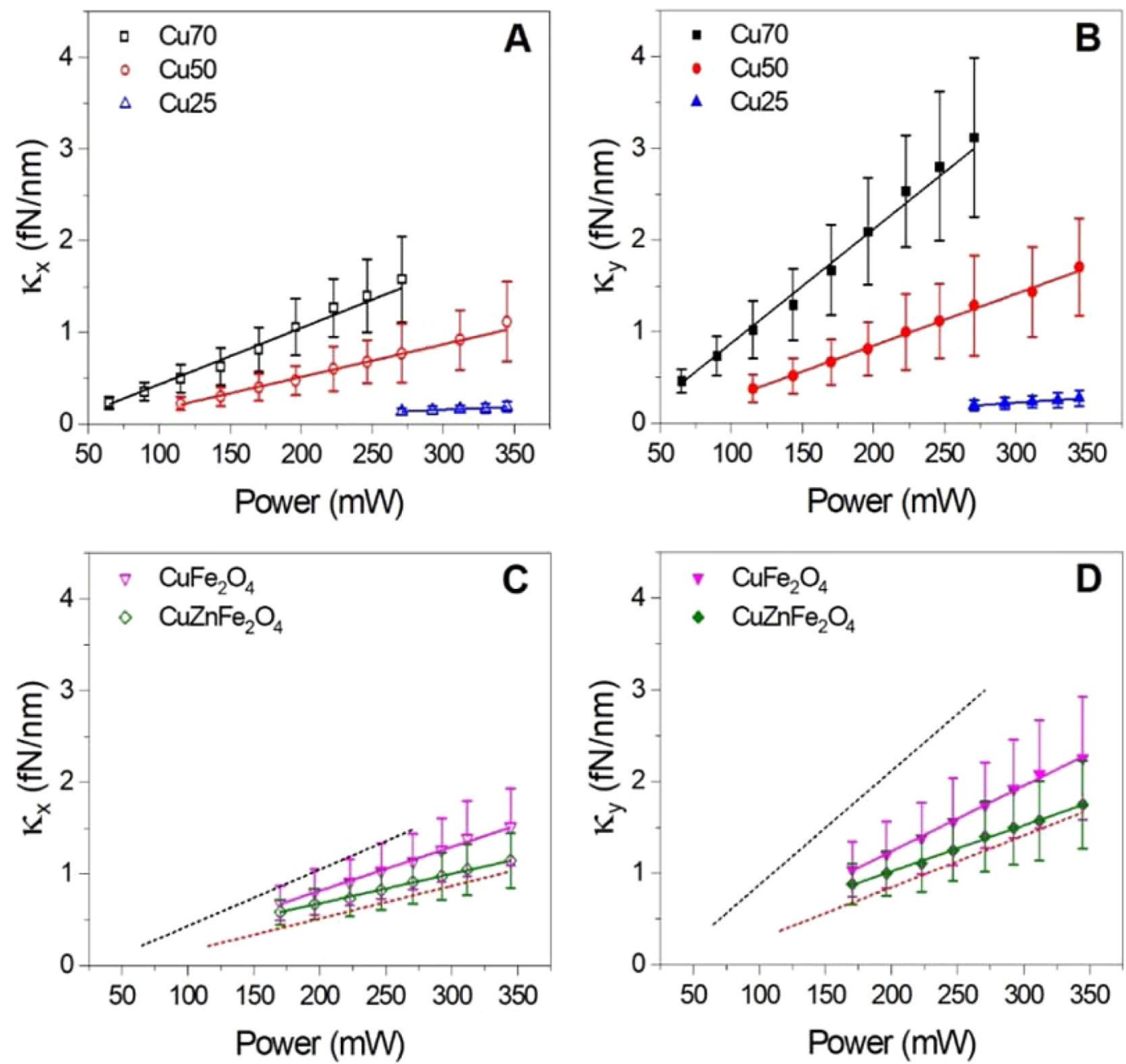

Figure 2. Trap stiffness for individual $\mathrm{Cu}, \mathrm{CuFe}_{2} \mathrm{O}_{4}$ and $\mathrm{CuZnFe}_{2} \mathrm{O}_{4}$ nanoparticles versus laser power. (A, B) Stiffness in a direction parallel (A) or perpendicular (B) to the laser's polarization for $70 \mathrm{~nm}$ (black squares), $50 \mathrm{~nm}$ (red circles), and $25 \mathrm{~nm}$ (blue triangles) CuNPs as a function of laser power at the sample plane. Lines show linear fits to data. (C,D) Same for $90 \mathrm{~nm} \mathrm{CuFe}{ }_{2} \mathrm{O}_{4}$ (magenta triangles) and $90 \mathrm{~nm} \mathrm{CuZnFe} \mathrm{O}_{4}$ (green diamonds) nanoparticles. Lines show linear fits to data. In (C,D) the linear fits for $70 \mathrm{~nm}$ and $50 \mathrm{~nm}$ CuNPs from $(\mathbf{A}, \mathbf{B})$ are plotted with dashed black and red lines, respectively, to allow for easier comparison between the trapping stiffness of solid CuNPs and $\mathrm{Cu}$ ferrites.

$$
\begin{gathered}
C_{a b s}=\frac{k}{\varepsilon_{0}} \alpha_{i} \\
C_{s c a t}=\frac{k^{4}}{6 \pi \varepsilon_{0}^{2}}|\alpha|^{2}
\end{gathered}
$$

where $k=2 \pi n_{m} / \lambda_{0}$, is the wavenumber, $n_{m}$ is the refractive index of the medium, and $\lambda_{0}$ the wavelength in vacuum.

As can be seen from Eq. 3, the gradient force is proportional to the real part of the particle's polarizability, $\alpha_{r}$, which can be found from Eqs. 5 and 6 if the cross sections are known. Equation 4 shows that the destabilizing scattering force is proportional to $C_{s c a t}+C_{a b s}$. In order to obtain stable $3 \mathrm{D}$ trapping, the gradient forces must balance or overcome the scattering force. The absorption cross section, $C_{a b s}$, provides information on the amount of energy absorbed by the particle and is directly related to the associated temperature increase.

To calculate $C_{s c a t}$ and $C_{a b s}$ for the massive CuNPs, we used finite element modeling (FEM) implemented in COMSOL software to solve the scattering problem in the frequency domain by numerically solving Maxwell's equations within a discretized space. This procedure has previously been employed to calculate optical properties of metallic nanoparticles and has been shown to provide results reproducing direct experimental measurements ${ }^{20,21}$. We used tetrahedral meshes with a mesh size of $1 / 10$ of the particle diameter. This is much below the threshold 

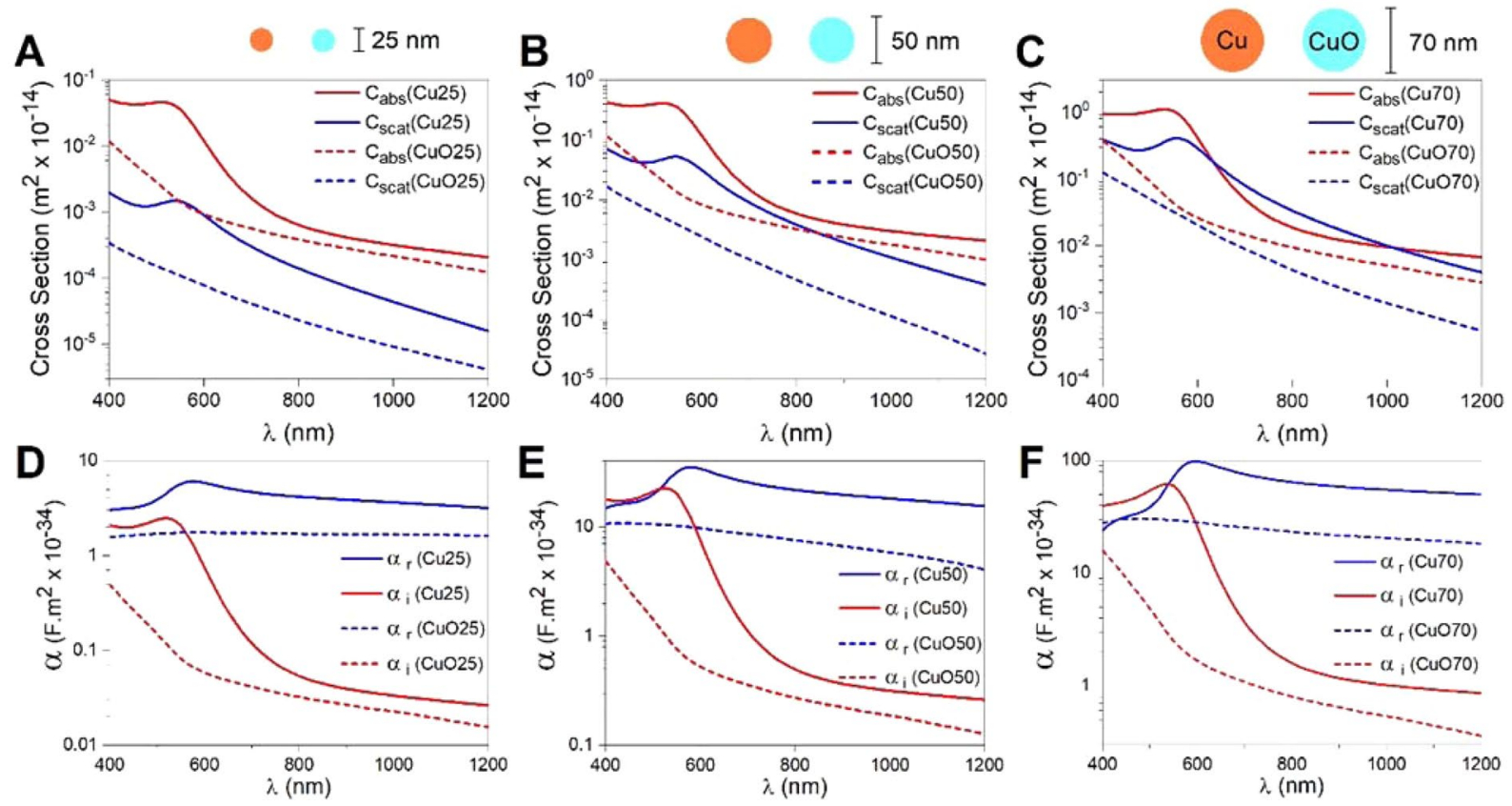

Figure 3. Absorption (red) and scattering (blue) cross-sections as a function of wavelength calculated by FEM for (A) $25 \mathrm{~nm}$, (B) $50 \mathrm{~nm}$, (C) $70 \mathrm{~nm}$ CuNPs (solid lines) and CuONPs (dashed lines). Real (blue) and imaginary (red) parts of polarizability versus wavelength for, (D) $25 \mathrm{~nm}$, (E) $50 \mathrm{~nm}$, (F) $70 \mathrm{~nm}$ CuNPs (solid lines) and CuONPs (dashed lines). Semi-log scales were employed to magnify the behaviour in the NIR region.

where further minimizing the mesh size does not change the results. By FEM we found the electric field, $\boldsymbol{E}$, satisfying the boundary condition

$$
\nabla \times \mu_{r}^{-1}(\nabla \times \boldsymbol{E})-k_{0}^{2}\left(\varepsilon_{r}-i \frac{\sigma}{\omega \varepsilon_{0}}\right) \boldsymbol{E}=0 .
$$

Here, $\boldsymbol{E}=\boldsymbol{E}_{\text {inc }}+\boldsymbol{E}_{\text {scat }}$, is a sum of the incoming and scattered electric fields. $\mu_{r}$ and $\varepsilon_{r}$ are the frequency dependent relative permeability and permittivities, respectively, $\omega$ is the angular frequency, $\sigma$ is conductivity and $k_{0}^{2}=\frac{\omega^{2}}{c^{2}}$. All optical constants and wavelength dependent $\mathrm{Cu}$ permittivities were deduced from fitting the Brendel-Bormann model to data ${ }^{22,23}$. For $\mathrm{CuO}$, the wavelength dependent permittivities were provided by Nanocomposix, Czech Republic.

From knowledge of $\boldsymbol{E}$ the absorption and scattering cross-sections can be calculated ${ }^{15}$

$$
\begin{gathered}
C_{a b s}=\frac{2}{c \varepsilon_{0}\left|\boldsymbol{E}_{i n c}\right|^{2}} \iiint q d V=\frac{2}{c \varepsilon_{0}\left|\boldsymbol{E}_{\text {inc }}\right|^{2}} \iiint \frac{1}{2} \operatorname{Re}\left(\boldsymbol{J} \cdot \boldsymbol{E}^{*}\right) d V \\
C_{\text {scat }}=\frac{2}{c \varepsilon_{0}\left|\boldsymbol{E}_{\text {inc }}\right|^{2}} \iint\left(\boldsymbol{n} \cdot \boldsymbol{S}_{\text {scat }}\right) d S=\frac{2}{c \varepsilon_{0}\left|\boldsymbol{E}_{\text {inc }}\right|^{2}} \iint \frac{1}{2}\left[n \cdot \operatorname{Re}\left(\boldsymbol{E}_{\text {scat }} \times \boldsymbol{H}_{\text {scat }}^{*}\right)\right] d S
\end{gathered}
$$

where $\boldsymbol{S}_{\text {scat }}=\frac{1}{2} \operatorname{Re}\left(\boldsymbol{E}_{\text {scat }} \times \boldsymbol{H}_{\text {scat }}^{*}\right)$ is the scattered Poynting vector along the normal vector, $\boldsymbol{n}$, in the outwards direction from the scatterer's surface that delimits the integral. The power loss density, $q$, is given by $q=\frac{1}{2} \operatorname{Re}\left(\boldsymbol{J} \cdot \boldsymbol{E}^{*}\right)$, where $\boldsymbol{J}=\sigma \boldsymbol{E}$ is the current density within the nanoparticle. Complex conjugates are denoted with an asterisk.

The calculated optical cross sections, $C_{a b s}$ and $C_{s c a t}$, are shown in Fig. 3A-C as a function of wavelength both for massive CuNPs and for fully oxidized CuNPs, CuONPs, of different sizes. For all wavelengths, both absorption and scattering cross sections are significantly higher for pure CuNPs than for CuONPs. Exact values for $C_{\text {scat }}$ and $C_{a b s}$ at the optical trapping wavelength, $1064 \mathrm{~nm}$, are given in Supporting Table S2.

The real and imaginary parts of the polarizability of CuNPs and CuONPs are shown in Fig. 3D-F. From Eq. (3) it is clear that $\alpha_{r}>0$ is a necessary condition for stable optical trapping. Hence, both CuNPs and CuONPs should be trappable within the whole wavelength window theoretically investigated, however, with CuNPs trapping more strongly than CuONPs, as experimentally observed for $\lambda=1064 \mathrm{~nm}$ (Fig. 2). Values for $\alpha_{r}$ and $\alpha_{i}$ at $\lambda=1064 \mathrm{~nm}$ are provided in Supporting Table S2.

As the gradient force, responsible for optical trapping, is directly proportional to $\alpha_{r}$ (see Eq. 3), we used the ratios of $\alpha_{r}$ and of $\kappa$ for different particle types to compare the calculated expectations for trapping strength to the measured values. This is not a perfect comparison as the scattering force will also contribute to the total force. However, in this regime the gradient force dominates the scattering force, therefore, we calculated the polarizability ratios $\alpha_{\mathrm{r}, \mathrm{Cu} 1} / \alpha_{\mathrm{r}, \mathrm{Cu} 2}$ and compared these to the corresponding ratios of the measured trap stiffnesses, $\kappa_{\mathrm{Cu} 1} / \kappa_{\mathrm{Cu} 2}$. 


\begin{tabular}{|l|l|l|l|}
\hline$\kappa_{\mathrm{y}}$ ratio & $\boldsymbol{\kappa}_{\mathrm{y}}$ ratio values & $\boldsymbol{\alpha}_{\boldsymbol{r}}$ ratio & $\boldsymbol{\alpha}_{\boldsymbol{r}}$ ratio values \\
\hline$\kappa_{y(\mathrm{Cu} 70)} / \kappa_{y(\mathrm{Cu} 50)}$ & $\mathbf{2 . 5}$ & $\alpha_{\mathrm{Cu} 70} / \alpha_{\mathrm{Cu} 50}$ & $\mathbf{3}$ \\
\hline$\kappa_{y(\mathrm{Cu} 70)} / \kappa_{y(\mathrm{Cu} 25)}$ & $\mathbf{1 6}$ & $\alpha_{\mathrm{Cu} 70} / \alpha_{\mathrm{Cu} 25}$ & $\mathbf{1 5}$ \\
\hline$\kappa_{y(\mathrm{Cu} 50)} / \kappa_{y(\mathrm{Cu} 25)}$ & $\mathbf{6 . 5}$ & $\alpha_{\mathrm{Cu} 50} / \alpha_{C u 25}$ & $\mathbf{5}$ \\
\hline
\end{tabular}

Table 1. Comparison of experimentally obtained trap stiffness ratios and the corresponding theoretically calculated ratios of the real part of the particles' polarizability, $\alpha_{r}$, for different solid CuNPs. The gradient force, responsible for optical trapping, is directly proportional to $\alpha_{r}$, therefore, the ratios of $\kappa$ and $\alpha_{r}$ for different particle sizes are expected to be similar.

These ratios, given in Table 1, demonstrate a good agreement between predicted increase of trapping stiffness as a function of particle size for massive CuNPs and the experimental findings.

Effect of oxidized shell on optical properties of CuNPs. To further explore the effects of oxidization, we performed FEM calculations of CuNPs with an oxidized shell of varying thickness. When Cu oxidizes, it swells, hence, in our simulations, we assumed that half the $\mathrm{CuO}$ shell thickness is taken from the CuNP due to the oxidation and the other half is caused by swelling of the oxide layer. For example, in Fig. 4A, we consider a CuNP which originally had $\mathrm{d}=70 \mathrm{~nm} ; 1 \mathrm{~nm}$ of this particle oxidizes, which causes an additional swelling of $1 \mathrm{~nm}$, hence, the total thickness of the oxide shell is $2 \mathrm{~nm}$. In consequence, the $\mathrm{Cu}$ core has $\mathrm{d}=68 \mathrm{~nm}$ and the overall diameter of the particle including the oxidized shell is $72 \mathrm{~nm}$. FEM results for three different core-shell sizes of $\mathrm{Cu}-\mathrm{Cu}_{\mathrm{x}} \mathrm{O}$ NPs are presented in Fig. 4. It is clear that core-shell NPs with thin $\mathrm{CuO}$ shells have optical cross-sections and polarizabilities of similar magnitudes as CuNPs of same size (Fig. 3). Moreover, thicker CuO layers lead to a significant change of optical properties.

Experimentally we have seen no evidence in the TEM images (as, e.g., shown in Fig. 1A-E) of a swelled oxide shell. Hence, if such an oxide shell was present, it was either rather thin or invisible in the TEM images.

As the gradient force, responsible for optical trapping, is directly proportional to $\alpha_{r}$ (cf. Eq. 3), trapping will not be possible at wavelengths where $\alpha_{r}$ is very small or negative. Figure $4 \mathrm{~F}$ illustrates such a scenario: for a shell thickness of $10 \mathrm{~nm}, \alpha_{\mathrm{r}}$ is negative around $\lambda=600 \mathrm{~nm}$ and such particles are hence not likely to be trappable at $600 \mathrm{~nm}$. The real part of the polarizability, $\alpha_{r}$, for pure CuNPs, is more than twice that of a fully oxidized NP (Table S2) and, hence, the effect of oxidation could significantly affect the trapping efficiency of the NPs. As the ratios of the calculated polarizabilities for massive and pure CuNPs compared well to the ratios of the experimentally determined trap stiffnesses (Table 1), it is consistent with our experimental measurements if the CuNPs acquired only a shallow oxide layer.

The altered optical trapping properties of CuNPs as a function of their oxidation imply that the degree of oxidation of CuNPs could, in principle, be explored by trapping the CuNPs at specific wavelengths. For instance, one could choose laser lines in the interval 600-700 nm where $\alpha_{r}$ changes significantly with the thickness of the oxide layer.

Plasmonic heating of irradiated CuNPs. Absorption of laser light by the trapped CuNPs will lead to heating of the particles with the temperature increase of a particle being proportional to $C_{a b s}$ and laser intensity ${ }^{28}$. This will naturally lead to an increase in the temperature of the media surrounding the particle which affects the viscosity of the medium and hence the motion of the particle. This phenomenon is known as Hot Brownian Motion $^{29,30}$ and has implications for the dynamics of the particle and, hence, for its optical trapping properties.

To estimate the temperature increase of an optically trapped CuNP we used FEM to theoretically predict its plasmonic heating in the laser trap. The temperature of an optically trapped plasmonic nanoparticle equilibrates within nanoseconds ${ }^{21}$, hence, at the timescales here considered, the temperature can be assumed constant across the particle and decays with distance to the particle's surface, $r$, as ${ }^{28,31}$

$$
\Delta T(r)=\frac{C_{a b s} I}{4 \pi K r}, r>R
$$

where $I$ is the trapping laser intensity, $R$ is the radius of nanoparticle, and $K$ is the thermal conductivity of surrounding medium (water). To find $C_{a b s}$ for each particle type we used FEM as described in a preceding section. Figure $5 \mathrm{~A}$ shows the temperature profiles for three different sizes of optically trapped CuNPs calculated via Eq. (5); for these calculations a constant laser power of $270 \mathrm{~mW}$ is used as this is the highest laser power used in the optical trapping experiments for all particle types (as shown in Fig. 2). Also, the temperature profile for a CuNP with an oxidized shell (core: $\mathrm{d}_{\mathrm{Cu}}=60 \mathrm{~nm}$ ), shell: $\mathrm{t}_{\mathrm{CuO}}=10 \mathrm{~nm}$ ) is presented (orange line in Fig. $5 \mathrm{~A}, \mathrm{~B}$ ). The larger the particle, the higher its temperature. Equation 10 predicts a linear relation between temperature increment and laser power, this is shown in Fig. 5B for the same NPs featured in Fig. 5A. Interestingly, the particle with an oxidized shell (orange lines in Fig. 5A,B, core $=60 \mathrm{~nm}$, shell: $\mathrm{t}=10 \mathrm{~nm}$ ) has a temperature that is higher than a similarly sized massive CuNP ( $80 \mathrm{~nm}$, grey).

Figure 5C shows the 2D temperature profile around an irradiated CuNP with an oxidized shell (core: $\mathrm{d}_{\mathrm{Cu}}=60 \mathrm{~nm}$, shell: $\mathrm{t}=10 \mathrm{~nm}$ ). Interestingly, varying the volume ratio between the $\mathrm{Cu}$ core and the oxidized shell can give rise to several hundreds of degrees of difference in the surface temperature at constant laser power.

In the calculations the particle is assumed to be located in the focus of a laser beam with a perfect Gaussian intensity profile. However, this is not a realistic experimental situation; inherent spherical aberration will 

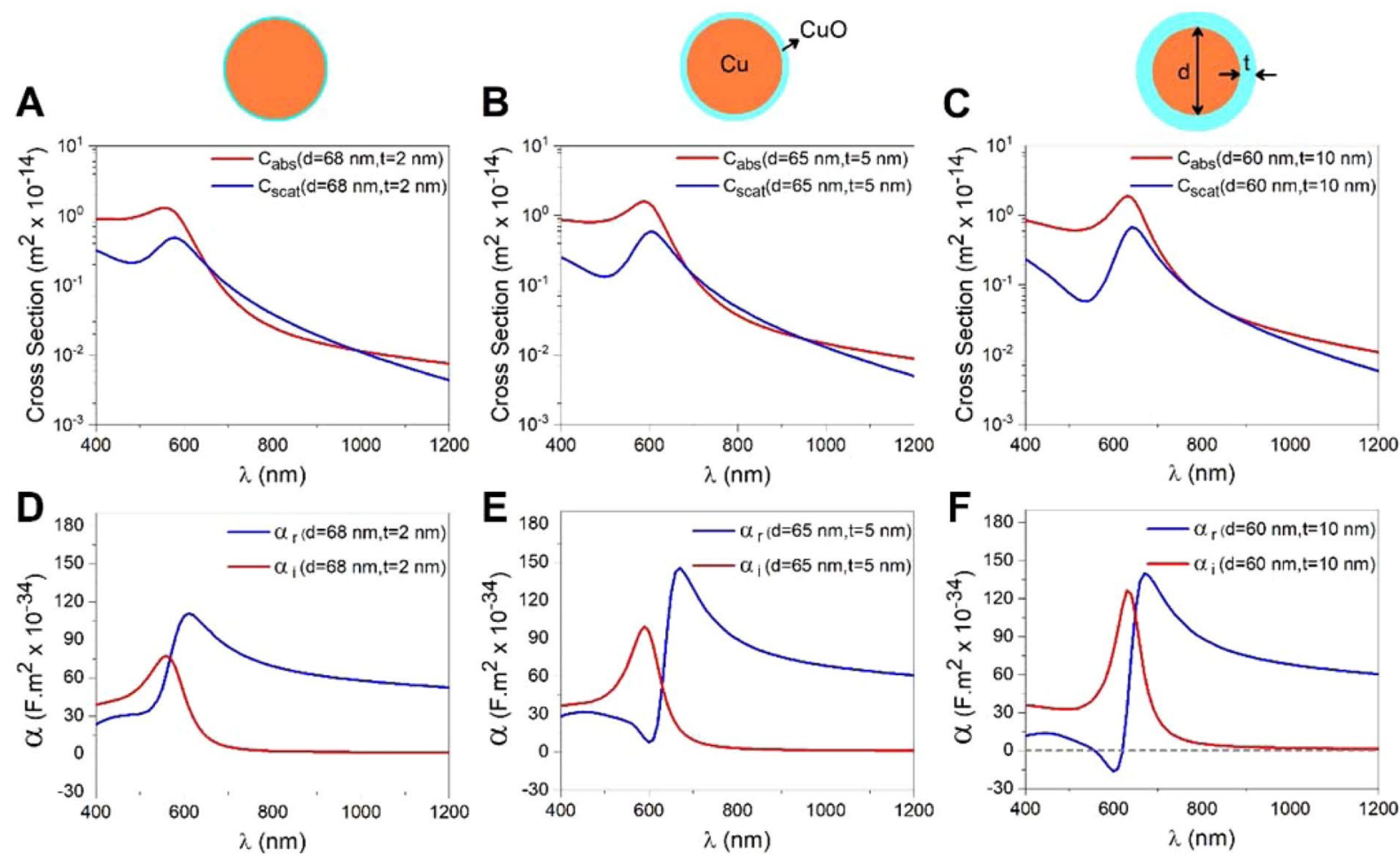

Figure 4. Absorption cross sections and polarization for CuNPs with an oxidized shell of varying thickness calculated by FEM. (A-C) Absorption (red), scattering (blue) cross-sections for CuNPs with an oxidized shell as a function of wavelength. The semi-log scales were employed to magnify the behaviour in the NIR region. (D-F) Real (blue) and imaginary (red) parts of polarizability versus wavelength. (A,D) Cu core: $\mathrm{d}=68 \mathrm{~nm}$, oxidized shell: $\mathrm{t}=2 \mathrm{~nm},(\mathbf{B}, \mathbf{E})$ Cu core; $\mathrm{d}=65 \mathrm{~nm}$, oxidized shell: $\mathrm{t}=5 \mathrm{~nm},(\mathbf{C}, \mathbf{F})$ Cu core: $\mathrm{d}=60 \mathrm{~nm}$, oxidized shell: $\mathrm{t}=10 \mathrm{~nm}$.

inevitably cause distortions of the intensity profile, distortions which are significant at the nanometer scale. Also, optically trapped metallic nanoparticles have been shown to stably trap at positions far from the focus, either below or above, depending in a non-trivial manner on the particle size, material and laser power ${ }^{32}$. These effects will cause the laser intensity at the position of the particle to be unpredictable and, probably, substantially different than in the theoretical situation.

Chemical reaction rates are sensitively dependent on temperature as quantified by the Arrhenius equation. Therefore, heating of CuNPs is likely to be of crucial importance for their catalytic activity ${ }^{4}$. In accordance with this, previous studies have demonstrated that the oxidation state of $\mathrm{Cu}$ can be modified by laser light ${ }^{33,34}$.

\section{Conclusions}

We demonstrated stable optical trapping of individual $\mathrm{Cu}, \mathrm{CuFe}_{2} \mathrm{O}_{4}$, and $\mathrm{CuZnFe}_{2} \mathrm{O}_{4}$ nanoparticles with diameters in the range of 25-90 $\mathrm{nm}$ using NIR light. We found that $\mathrm{Cu}$-ferrites had a weaker interaction with the optical trap compared to CuNPs of similar sizes, which can be attributed to the ionic character of the oxides compared to the metallic character of pure $\mathrm{Cu}$ particles. By finite element modelling we calculated the optical properties of CuNPs and their oxides and showed that the presence of an oxidized shell can significantly change the optical properties of CuNPs. By tuning the ratio of the $\mathrm{Cu}$ core and the thickness of the oxidized shell one can obtain a situation where the real part of the polarizability becomes negative at certain wavelengths, thus rendering the particles non-trappable. Our experimental optical trapping measurements of solid CuNPs in water, however, indicated only minor to no oxidation of the surface. Due to the plasmonic nature of CuNPs, they are expected to heat significantly in the optical trap. Our theoretical calculations show that, if trapped in the focus of the laser beam, temperature increases up to hundreds of degrees Celsius are to be expected, with the exact value being crucially dependent on the particle's size, oxidation state and position. This temperature increase is likely to affect CuNPs' catalytic properties. Also, heating might cause ablation of the oxide layer and restore the physicochemical properties of the pure CuNP. Hence, laser induced heating may allow for remote control over the particle's chemical and catalytic properties.

\section{Methods}

Optics and data acquisition. An inverted microscope (LEICA DMIRB HC) was used to integrate the optical trap, based on a CW Nd:YVO 4 laser $(\lambda=1064 \mathrm{~nm}, 5 \mathrm{~W}$, Spectra Physics). The laser was focused by an oil immersion objective (HCX, PL, APO, $100 \times /$ N.A. $=1.4$ ). The immersion oil had an index of refraction of $n=1.54$, ensuring minimum spherical aberration at the sample plane, located approximately at $5 \mu \mathrm{m}$ from the chamber's lower surface. A quadrant photodiode (QPD) (S5981, Hamamatsu) was placed in the conjugate plane of the 

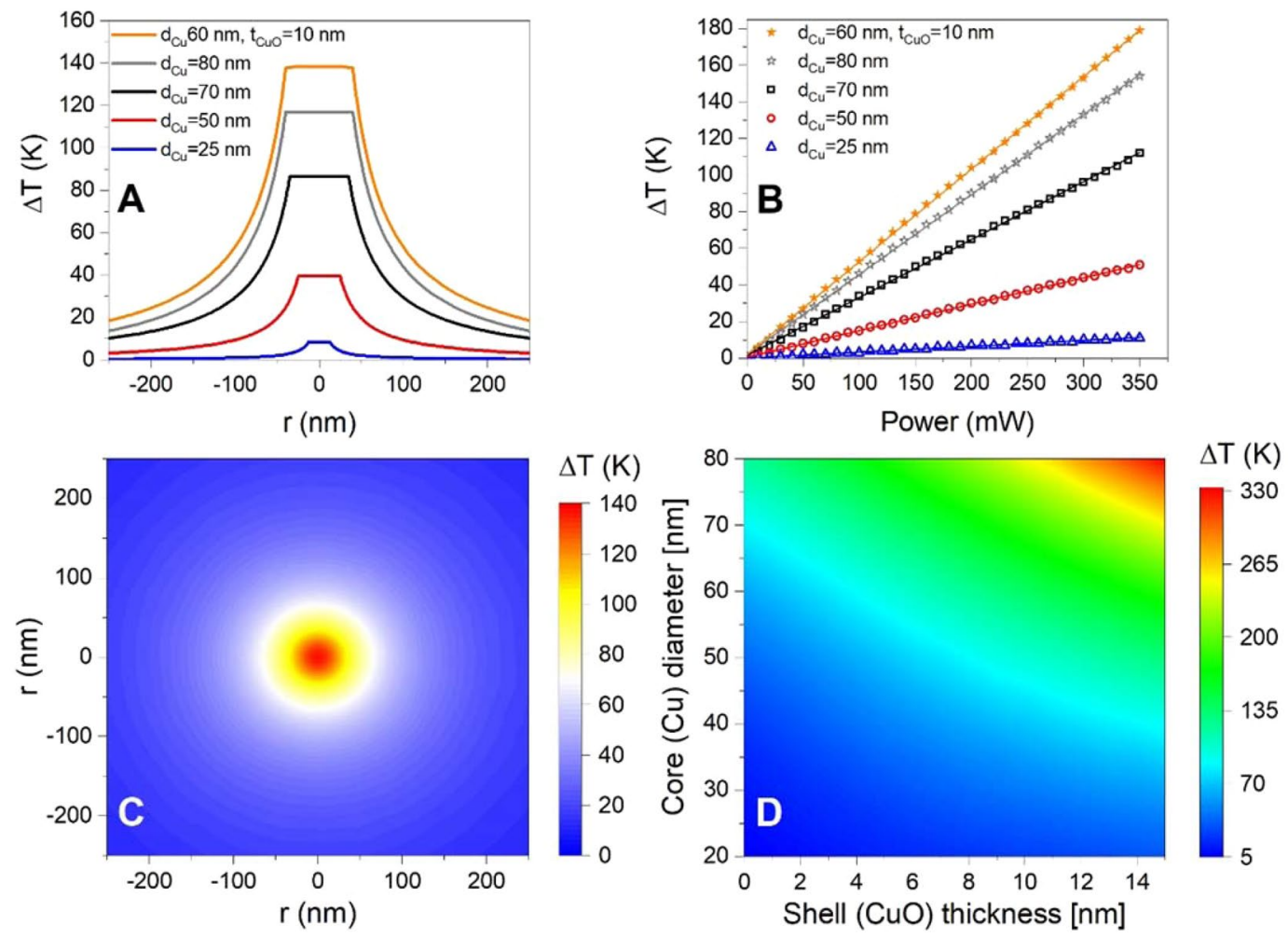

Figure 5. Theoretical calculation of plasmonic heating of trapped solid CuNPs and CuNPs with an oxidized shell. (A) Temperature profile around $25 \mathrm{~nm}$ (blue), $50 \mathrm{~nm}$ (red), $70 \mathrm{~nm}$ (black), and $80 \mathrm{~nm}$ (grey) solid CuNPs, as well as for a CuNP with an oxidized shell (core: $\mathrm{d}=60 \mathrm{~nm}$, shell: $\mathrm{t}=10 \mathrm{~nm}$ ) under the same conditions as in the optical trapping experiments. (B) Temperature increase as a function of laser power for the same particles as shown in (A). (C) $2 \mathrm{D}$ heat profile around a CuNP with an oxidized shell (core: $\mathrm{d}_{\mathrm{Cu}}=60 \mathrm{~nm}$, shell: $\mathrm{t}=10 \mathrm{~nm}$ ). (D) Temperature increase for CuNPs with an oxidized shell for varying sizes of the diameter of the core and the thickness of the oxidized shell. For these calculations, $\lambda=1064 \mathrm{~nm}$ and $\mathrm{P}=270 \mathrm{~mW}$.

condenser's back focal plane to monitor the trapped particle position. To visualize the trapped particles, a CCD camera ( $25 \mathrm{~Hz}$ frame rate, Sony XC-EI50) was used to detect the backscattered light. When a particle was trapped, we monitored a significant change in the QPD signal; the amplitude of the time series, signifying the variance of the positions visited, increased to a steady value upon successful trapping. Should a second particle enter the trap, the signal on the QPD would again change as described in ref. ${ }^{16}$. Also, we performed real-time calculations of the power spectrum of the QPD signal and the characteristic Lorentzian spectrum as displayed in Fig. 1G would only emerge when a particle was successfully trapped. A custom-made Labview program was used for processing the time series acquired by the QPD.

Sample preparation. Dry nanopowdered Cu NPs with mean diameters of $25 \mathrm{~nm}$ (Cu25), $50 \mathrm{~nm}(\mathrm{Cu} 50)$ and $70 \mathrm{~nm}(\mathrm{Cu} 70)$ as well as $90 \mathrm{~nm}$ in diameter $\mathrm{CuFe}_{2} \mathrm{O}_{4}$ and $\mathrm{CuZnFe}_{2} \mathrm{O}_{4} \mathrm{NPs}$ were purchased from mkNano (Canada). A small portion of the powders (below $1 \mathrm{mg}$ ) was suspended in $1.5 \mathrm{ml}$ of ultrapure milliQ water and sonicated for $\sim 20$ minutes and then filtered through $100 \mathrm{~nm}$ pore diameter membranes to reduce the possible presence of clusters in the trapping chambers. Perfusion chambers were built by using a microscope slide and a cover glass separated by double-sided scotch tape. The volume of the chamber was completely filled with the diluted nano-suspensions.

TEM imaging. The TEM images of the different particle types shown in Fig. 1A-E were obtained using a transmission electron microscope (Philips CM-12 (120 kV) and Philips EM-420 (120 kV) located at the University of Copenhagen.

Received: 27 March 2019; Accepted: 28 December 2019;

Published online: 27 January 2020

\section{References}

1. McNamara, K. \& Tofail, S. A. Nanosystems: the use of nanoalloys, metallic, bimetallic, and magnetic nanoparticles in biomedical applications. Phys. Chem. Chem Phys 17, 27981-27995, https://doi.org/10.1039/c5cp00831j (2015).

2. Scholl, J. A., Koh, A. L. \& Dionne, J. A. Quantum plasmon resonances of individual metallic nanoparticles. Nature 483, 421-427, https://doi.org/10.1038/nature10904 (2012).

3. Jiang, Z., Le, N. D. B., Gupta, A. \& Rotello, V. M. Cell surface-based sensing with metallic nanoparticles. Chem. Soc. Rev. 44, 4264-4274, https://doi.org/10.1039/c4cs00387j (2015). 
4. Baffou, G. \& Quidant, R. Nanoplasmonics for chemistry. Chem. Soc. Rev. 43, 3898-3907, https://doi.org/10.1039/c3cs60364d (2014).

5. Gawande, M. B. et al. $\mathrm{Cu}$ and Cu-Based Nanoparticles: Synthesis and Applications in Catalysis. Chem. Rev. 116, 3722-3811, https:// doi.org/10.1021/acs.chemrev.5b00482 (2016).

6. Wang, C., Ling, L., Yao, Y. \& Song, Q. One-step synthesis of fluorescent smart thermo-responsive copper clusters: A potential nanothermometer in living cells. Nano Res. 8, 1975-1986, https://doi.org/10.1007/s12274-015-0707-0 (2015).

7. Hu, L. et al. Copper nanoclusters as peroxidase mimetics and their applications to $\mathrm{H}_{2} \mathrm{O}_{2}$ and glucose detection. Anal. Chim. Acta 762, 83-86, https://doi.org/10.1016/j.aca.2012.11.056 (2013).

8. Wang, Y. et al. Integrated amplified aptasensor with in-situ precise preparation of copper nanoclusters for ultrasensitive electrochemical detection of microRNA 21. Biosens. Bioelectron. 98, 386-391, https://doi.org/10.1016/j.bios.2017.07.009 (2017).

9. Zhang, X. et al. Effects of copper nanoparticles in porous $\mathrm{TiO} 2$ coatings on bacterial resistance and cytocompatibility of osteoblasts and endothelial cells. Mater. Sci. Eng. C. Mater Biol. Appl. 82, 110-120, https://doi.org/10.1016/j.msec.2017.08.061 (2018).

10. Xu, L., Liang, H. W., Yang, Y. \& Yu, S. H. Stability and Reactivity: Positive and Negative Aspects for Nanoparticle Processing. Chem. Rev. 118, 3209-3250, https://doi.org/10.1021/acs.chemrev.7b00208 (2018).

11. Ahamed, M., Akhtar, M. J., Alhadlaq, H. A. \& Alshamsan, A. Copper ferrite nanoparticle-induced cytotoxicity and oxidative stress in human breast cancer MCF-7 cells. Colloids Surf. B Biointerfaces 142, 46-54, https://doi.org/10.1016/j.colsurfb.2016.02.043 (2016).

12. Alzahrani, K. E. et al. Antibacterial activity of trimetal (CuZnFe) oxide nanoparticles. Int. J. Nanomed. 13, 77-87, https://doi. org/10.2147/IJN.S154218 (2018).

13. Wang, A. et al. Plasmon mediated Fe-O in an octahedral site of cuprospinel by $\mathrm{Cu}$ NPs for photocatalytic hydrogen evolution. Nanoscale 9, 15760-15765, https://doi.org/10.1039/c7nr06217f (2017).

14. Ashkin, A., Dziedzic, J. M., Bjorkholm, J. E. \& Chu, S. Observation of a single-beam gradient force optical trap for dielectric particles. Opt. Lett. 11, 288, https://doi.org/10.1364/ol.11.000288 (1986).

15. Svoboda, K. \& Block, S. M. Optical trapping of metallic Rayleigh particles. Opt. Lett. 19, 930-932 (1994).

16. Poul Martin, H., Vikram Kjoller, B., Niels, H. \& Lene, O. Expanding the Optical Trapping Range of Gold Nanoparticles. Nano Lett. 5, 1937-1942 (2005).

17. Lana Bosanac, T. A., Bendix, P. M. \& Oddershede, L. B. Efficient Optical Trapping and Visualization of Silver Nanoparticles. Nano Lett. 8, 1486-1491 (2008).

18. Samadi, A., Bendix, P. M. \& Oddershede, L. B. Optical manipulation of individual strongly absorbing platinum nanoparticles. Nanoscale 9, 18449-18455, https://doi.org/10.1039/c7nr07374g (2017).

19. Bendix, P. M., Reihani, S. N. S. \& Oddershede, L. B. Direct Measurements ofHeating by Electromagnetically Trapped Gold Nanoparticles on Supported Lipid Bilayers. ACS Nano 4, 2256-2262 (2010).

20. Ma, H., Tian, P., Pello, J., Bendix, P. M. \& Oddershede, L. B. Heat generation by irradiated complex composite nanostructures. Nano Lett. 14, 612-619, https://doi.org/10.1021/nl403798j (2014).

21. Samadi, A. et al. Platinum nanoparticles: a non-toxic, effective and thermally stable alternative plasmonic material for cancer therapy and bioengineering. Nanoscale 10, 9097-9107, https://doi.org/10.1039/c8nr02275e (2018)

22. Durdevic, L., Robert, H. M. L., Wattellier, B., Monneret, S. \& Baffou, G. Microscale Temperature Shaping Using Spatial Light Modulation on Gold Nanoparticles. Sci. Rep. 9, 4644, https://doi.org/10.1038/s41598-019-40382-3 (2019).

23. Baffou, G. et al. Photoinduced heating of nanoparticle arrays. ACS Nano 7, 6478-6488, https://doi.org/10.1021/nn401924n (2013).

24. Friese, M. E. J., Enger, J., RubinszteinDunlop, H. \& Heckenberg, N. R. Optical angular-momentum transfer to trapped absorbing particles. Phys. Rev. A 54, 1593-1596, https://doi.org/10.1103/PhysRevA.54.1593 (1996)

25. Hansen, P. M., Tolić-Nørrelykke, I. M., Flyvbjerg, H. \& Berg-Sørensen, K. tweezercalib 2.0: Faster version of MatLab package for precise calibration of optical tweezers. Computer Phys. Commun. 174, 518-520, https://doi.org/10.1016/j.cpc.2005.11.007 (2006).

26. Albaladejo, S., Marques, M. I., Laroche, M. \& Saenz, J. J. Scattering forces from the curl of the spin angular momentum of a light field. Phys. Rev. Lett. 102, 113602, https://doi.org/10.1103/PhysRevLett.102.113602 (2009).

27. Lehmuskero, A., Johansson, P., Rubinsztein-Dunlop, H., Tong, L. \& Kall, M. Laser trapping of colloidal metal nanoparticles. ACS Nano 9, 3453-3469, https://doi.org/10.1021/acsnano.5b00286 (2015).

28. Jauffred, L., Samadi, A., Klingberg, H., Bendix, P. M. \& Oddershede, L. B. Plasmonic Heating of Nanostructures. Chem. Rev. 119, 8087-8130, https://doi.org/10.1021/acs.chemrev.8b00738 (2019).

29. Rings, D., Schachoff, R., Selmke, M., Cichos, F. \& Kroy, K. Hot brownian motion. Phys. Rev. Lett. 105, 090604, https://doi. org/10.1103/PhysRevLett.105.090604 (2010).

30. Rings, D., Selmke, M., Cichos, F. \& Kroy, K. Theory of Hot Brownian Motion. Soft Matter 7, 3441-3452 (2011).

31. Goldenberg, H. \& Tranter, C. J. Heat Flow in an Infinite Medium Heated by a Sphere. Br. J. Appl. Phys. 3, 296-298 (1952).

32. Kyrsting, A., Bendix, P. M. \& Oddershede, L. B. Mapping 3D focal intensity exposes the stable trapping positions of single nanoparticles. Nano Lett. 13, 31-35, https://doi.org/10.1021/nl3032263 (2013).

33. Marimuthu, A., Zhang, J. \& Linic, S. Tuning selectivity in propylene epoxidation by plasmon mediated photo-switching of $\mathrm{Cu}$ oxidation state. Science 339, 1590-1593, https://doi.org/10.1126/science.1231631 (2013).

34. Watanabe, K., Menzel, D., Nilius, N. \& Freund, H. J. Photochemistry on metal nanoparticles. Chem. Rev. 106, 4301-4320, https:// doi.org/10.1021/cr050167g (2006)

\section{Acknowledgements}

This work was supported by the Novo Nordisk Foundation, grant NNFOC150011361, the Danish National Research Foundation, grant DNRF116, the Danish Council for Independent Research DFF, grant number 418100196 and by the Spanish Ministerio de Economia y Competitividad under Project CTQ2014-56058P (including an FPI grant) and Project CTQ2017-82137.

\section{Author contributions}

P.P., A.S., P.M.B., J.J.L. and L.B.O. proposed and designed this study. Experiments and analyses were performed by P.P. and A.S., P.P. carried out the optical trapping experiments and A.S. the finite element simulations. All authors were involved in discussion of the results and in writing the paper.

\section{Competing interests}

The authors declare no competing interests.

\section{Additional information}

Supplementary information is available for this paper at https://doi.org/10.1038/s41598-020-57650-2.

Correspondence and requests for materials should be addressed to L.B.O. 
Reprints and permissions information is available at www.nature.com/reprints.

Publisher's note Springer Nature remains neutral with regard to jurisdictional claims in published maps and institutional affiliations.

(c) (i) Open Access This article is licensed under a Creative Commons Attribution 4.0 International License, which permits use, sharing, adaptation, distribution and reproduction in any medium or format, as long as you give appropriate credit to the original author(s) and the source, provide a link to the Creative Commons license, and indicate if changes were made. The images or other third party material in this article are included in the article's Creative Commons license, unless indicated otherwise in a credit line to the material. If material is not included in the article's Creative Commons license and your intended use is not permitted by statutory regulation or exceeds the permitted use, you will need to obtain permission directly from the copyright holder. To view a copy of this license, visit http://creativecommons.org/licenses/by/4.0/.

(C) The Author(s) 2020 